\title{
Thermal Power Regulation System for Pulsed Fusion Reactor
}

\author{
Nobuyuki Inoue, Yuichi Ogawa and Zensho Yoshida \\ Department of Nuclear Engineering, Faculty of Engineering, \\ University of Tokyo, Tokyo 113.
}

(Received March 26, 1992/Revised Manuscript Received May 28, 1992)

\begin{abstract}
A new method of generating continuous electrical power using a pulsed DT tokamak fusion reactor is described. The system consists of an electrolysis-cell hydrogen producer and pulsed fusion reactors so as to keep continuous electric power output level by regulating thermal power flow in the reactor coolant circuit. It allso protects reactor structures and turbine blades from repeated thermal stresses caused by pulsed operations or by disruptive instabilities. In case of the reactor with a thermal output power of $3 \mathrm{GW}$ and with an operation as long as $20 \mathrm{~h}$ with a dwell time of $5 \mathrm{~min}$, the circulation power necessary for generating hydrogen fuels used for regulating thermal power level is only 15.3 MW. A sufficient amount of deuterium fuels is produced as by-products.

Keywords:

pulsed fusion reactor, tokamak, hydrogen, electrolysis, deuterium fuel, thermal power regulation, circulation power, energy multiplication factor,
\end{abstract}

\section{Introduction}

An ultra-long-pulse tokamak reactor IDLT $^{1)}$ (Inductively Operated Day-Long Tokamak) has numerous advantages such as no current drive system, high energy multiplication factor, low plasma current, small and short-pulse heating power for ignition, simple structure due to the absence of current drive system, and so forth. While its major disadvantage is the periodic shutdown of the reactor because of recharging of the Ohmic transformer. This is inevitable as long as the inductive current drive is adopted.

Cyclic operations of the reactor induce mechanical and thermal fatigue problems on the reactor structures such as the vacuum chamber, the blanket, and so on. Especially, that on the turbine blades of the electric power generator is most serious ${ }^{2}$. The IDLT reactor experiences about $10^{4}$ times of thermal stresses during its 30 -year lifetime. Although $10^{4}$ times of the heat cycle is estimated to be tolerable for large scale fusion reactors with $1 \mathrm{GW}$ of electric power output ${ }^{3)}$, the effect of daily cutoff of high power electricity on the power supply network is fatal. To compensate fusion power during a dwell period, some kinds of energy storage system are taken into account ${ }^{2)}$.

Here we propose a new method for generating continuous electric power output using a pulsed reactor and avoiding serious thermal fatigues on the reactor structures. The next section describes the details of proposed system and its function, and the Section 3 is devoted to discussions and summaries. 


\section{Heat Compensation System during Reactor Shutdown}

Figure 1 shows the flow circuit diagram of the reactor coolant. A boiler with hydrogen gas burners is inserted between the blanket and the turbine generator. Hydrogen gases are supplied from a high pressure reservoir which accumulates hydrogen gases produced in the water electrolysis cell during the operation time of the reactor. Deuterium fuel of the reactor is produced simultaneously as a by-product.

Prior to the scheduled cutoff of the fusion power, the burner is turned on so that the continuous flow of the thermal energy is supplied to the turbine generator and the first-wall blanket system. In order to avoid the thermal stresses, it takes hours to start up the burner-boiler system of the conventional steam power generator. In the case of the present system, however, the hot coolant is flowing in advance in the cooling loop, and thereby the required warming-up time of the hydrogen burner-boiler system can be shortened. Thus the damage of turbine blades and the reactor structures due to thermal fatigue can be minimized.

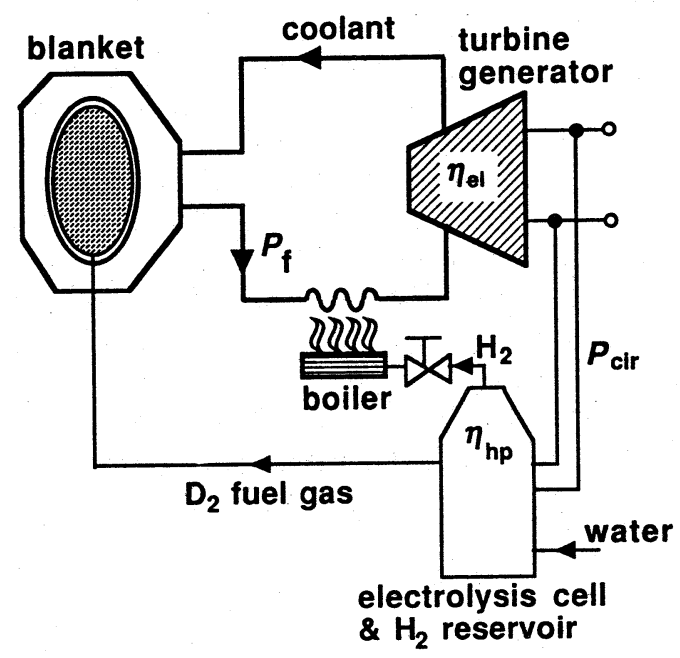

Fig. 1

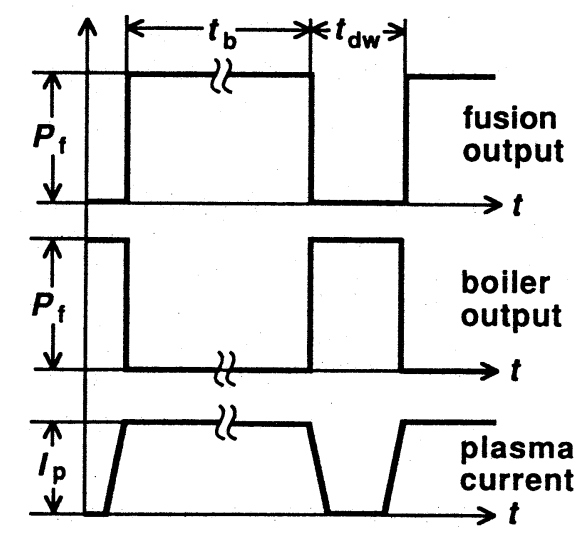

Fig. 2 
Figure 2 shows the time sequence of the fusion thermal power output, the thermal power output from the boiler, and the plasma current of the IDLT reactor. We set the fusion burning time of the core plasma $t_{\mathrm{b}}$, and the reactor dwell time $t_{\mathrm{dw}}$. The actual time evolution of the fusion power output will be somewhat more complicated than the stepwise change shown in the figure. The boiler output power is adjusted to be equal to the fusion power output $P_{\mathrm{f}}$ to compensate the power cutoff due to reactor shutdown.

The required integrated output energy transferred to the coolant from the boiler is

$$
W_{\mathrm{dw}}=P_{\mathrm{f}} t_{\mathrm{dw}} \text {. }
$$

This energy is produced by burning hydrogen gases, which are generated by electrolysis cells. The electric power feedback from the turbine generator output for producing hydrogen gases is given by

$$
P_{\mathrm{cir}}=\frac{1}{\eta_{\mathrm{hp}}} \frac{W_{\mathrm{dw}}}{t_{\mathrm{b}}+t_{\mathrm{dw}}},
$$

where $\eta_{\mathrm{hp}}$ is the energy efficiency of hydrogen production in the electrolysis cells. We define the energy multiplication factor of the reactor $Q^{*}$ by

$$
Q^{*}=\frac{P_{\mathrm{f}}}{P_{\mathrm{cir}}}=\eta_{\mathrm{hp}} \frac{t_{\mathrm{b}}+t_{\mathrm{dw}}}{t_{\mathrm{dw}}},
$$

which is related with the conventional energy multiplication factor $Q$ as

$$
Q\left(=\frac{P_{\mathrm{f}}}{P_{\text {input }}}\right)=\frac{P_{\mathrm{f}}}{\eta_{\text {conv }} P_{\text {cir }}}=\frac{Q^{*}}{\eta_{\text {conv }}},
$$

where $\eta_{\text {conv }}$ is the conversion efficiency of the circulating electric power to the power for heating/current drives. Both large $\eta_{\mathrm{hp}}$ and large $t_{\mathrm{b}} / t_{\mathrm{dw}}$ are required for high $Q$ reactors.

Since the energy produced by burning hydrogen gases is $285.8 \mathrm{~kJ} / \mathrm{mol}$, the amount of hydrogen fuels necessary for producing $W_{\mathrm{dw}}$ is given by

$$
M\left(\mathrm{H}_{2}\right)=3.5 \times 10^{-6} W_{\mathrm{dw}} \text { mol. }
$$

The total charge necessary for producing $M\left(\mathrm{H}_{2}\right)$ mol of hydrogen gases in the electrolysis cells is given by

$$
q\left(\mathrm{H}_{2}\right)=2 N_{\mathrm{A}} e M\left(\mathrm{H}_{2}\right) .
$$

where $N_{\mathrm{A}}\left(=6.02 \times 10^{23}\right.$ atom $\left./ \mathrm{mol}\right)$ is the Abogadro number and $e\left(=1.6021 \times 10^{-19} \mathrm{C}\right)$ is the charge of a proton.

Let us assume that $P_{\mathrm{f}}=3 \mathrm{GW}, t_{\mathrm{dw}}=5 \mathrm{~min}=300 \mathrm{~s}$, and $t_{\mathrm{b}}=20 \mathrm{~h}=72,000 \mathrm{~s}$. Then from Eq. (1), $W_{\mathrm{dw}}=9 \times 10^{11} \mathrm{~J}$, and from Eq. (6), $q\left(\mathrm{H}_{2}\right)=6.1 \times 10^{11} \mathrm{C}$. The necessary current for producing hydrogen gases during the fusion burning period and dwell time is $q\left(\mathrm{H}_{2}\right) /\left(t_{\mathrm{b}}+t_{\mathrm{dw}}\right)=8.4$ MA. The typical voltage and current density between electrodes of the electrolysis cells for industrial use are $1.8 \mathrm{~V}$ and $500 \mathrm{Am}^{-2}$, respectively. Then the total area of the electrode surfaces becomes $1.7 \times 10^{4} \mathrm{~m}^{2}=(130 \mathrm{~m})^{2}$, and the power consumed in the electrolysis cell is 15.1 MW. This is equivalent that the efficiency of hydrogen production $\eta_{\mathrm{hp}}$ is 0.82 from Eq. (3). Electrodes with a huge surface area can be divided into many smaller pieces to construct a compact stack, while keeping the necessary total area.

The power multiplication factor $Q^{*}$ is $199(=3 \mathrm{GW} / 15.1 \mathrm{MW})$ in the present case. The plant efficiency of the fusion power plant is defined as the net electric power output $P_{\mathrm{N}}$ divided by the fusion power of the reactor, and in the present case it becomes

$$
\eta=\frac{P_{\mathrm{N}}}{P_{\mathrm{f}}}=\frac{\eta_{\mathrm{el}} P_{\mathrm{f}}-P_{\mathrm{cir}}}{P_{\mathrm{f}}}=\eta_{\mathrm{el}}\left(1-\frac{1}{\eta_{\mathrm{el}} Q^{*}}\right),
$$

where $\eta_{\mathrm{el}}$ is the conversion efficiency of the turbine generator from the thermal power to the electric power, and we assumed that both the heating efficiency of the hydrogen burner and the 
energy multiplication factor of the blanket are unity. The thermal-to-electric power conversion efficiency is typically $1 / 3$. Then with the use of the above value of the power multiplication factor $\left(Q^{*}=199\right)$ we can put $1 \ll \eta_{\mathrm{el}} Q^{*}$, and we conclude that the plant efficiency is sufficiently close to the power conversion efficiency of the turbine generator.

If we admit $Q^{*}=25$ (which corresponds to $Q=50$ as assumed in the SSTR reactor design ${ }^{4)}$ taking $\eta_{\text {conv }}=0.5$ ), we obtain the burning period $t_{\mathrm{b}}=2.5 \mathrm{~h}$ for $\eta_{\mathrm{hp}}=0.82$ and $t_{\mathrm{dw}}=5 \mathrm{~min}$. In this case the size of Ohmic transformer reduces as well as the rector cost, the rector structure, however, must be designed to tolerate mechanical stresses induced by $10^{5}$ times of the cyclic operation.

The amount of deuterium fuels burnt through the DT fusion reaction for generating 3 $\mathrm{GW}$ of thermal power for $20 \mathrm{~h}$ is $64 \mathrm{~mol}$. While that of hydrogen gases generated by the electrolysis is $3.15 \times 10^{6} \mathrm{~mol}$ from Eq. (4). Since the natural content of the deuterium is $0.015 \%$ of the total hydrogen isotopes, the amount of deuterium gases generated simultaneously in the electrolysis cell is $473 \mathrm{~mol}$. Hence a sufficient amount of deuterium fuels is generated by the electrolysis during the burning period.

\section{Discussion and Summary}

The thermal power regulation system described here is based on the mature technologies, i.e., the electrolysis technology and the water boiler technology. The electrolysis technology is quite important from the viewpoint that the petroleum, which is now used as a portable fuel in many of the conveyances such as ships and air crafts, will be replaced by the pollution-free hydrogen fuels in the future. Fusion reactors combined with the electrolysis cell will presumably become a main generator of hydrogen fuels. Therefore, it is worth to start researches and developments of the power generation system described here as soon as possible. With the use of the thermal power regulation system proposed above, we may start designing the fusion power plant without waiting the establishment of the plasma current drive technology. That will make the earlier realization of the fusion power plant.

The thermal power regulation system will also be useful on the occurrence of a sudden tokamak plasma shutdown caused by unexpected disruptive instabilities, because the serious thermal stresses will be alleviated with this system. Supplemental hydrogen gas generators and the reservoir system are necessary for this purpose.

In summary, a new method for converting the pulsed fusion power output to the continuous electric power output is proposed. It produces a sufficient amount of deuterium fuels for DT reactors as a by-product, and can avoid serious thermal fatigues on the reactor structures and the turbine blades caused by short time shutdown of the fusion power production. The necessary circulation power for generating an enough amount of hydrogen gases is sufficiently small compared with the reactor output power.

\section{REFERENCES}

1) N. Inoue and Y. Ogawa: Kakuyugo Kenkyu 67 (1992) 344.

2) D.A. Ehst, Y. Cha, A.M. Hassanein, S. Majumdar, B. Misra and H.C. Stevens: Nuclear Engineering and Design/Fusion 2 (1985) 305.

3) D.A. Ehst, J.N. Brooks, K. Evans, Jr. and S. Kim: Nuclear Engineering and Design/Fusion 2 (1985) 319.

4) Y. Seki, M. Kikuchi, T. Ando et al.: Plasma Physics and Controlled Nuclear Fusion Research 1990 (Proc. 13th Int. Conf. Washington D.C., 1990), IAEA, Vienna (1991) Vol. 3, 473. 